\title{
Improvement on Response Performance of Min-Max Modular Classifier by Symmetric Module Selection*
}

\author{
Hai Zhao and Baoliang Lu \\ Department of Computer Science and Engineering, Shanghai Jiao Tong University \\ 1954 Huashan Rd., Shanghai 200030, China \\ \{zhaohai, blu\}@cs.sjtu.edu.cn
}

\begin{abstract}
This paper aims to improve the response performance of min-max modular classifier by a module selection policy for two-class classification during recognition. We propose an efficient base classifier selection algorithm. We show that the quadratic complexity of original min-max modular classifier can fall onto the level of linear complexity in the number of base-classifier modules for each input sample under presented selection scheme. The experimental results indicate the effectiveness of our algorithm and verify our theoretical analysis.
\end{abstract}

\section{Introduction}

Two-class classification is one kind of basic classification problem. Many essential classification schemes often start from binary classifier and then adapt to multiclass classifiers. Therefore, an improvement on two-class problem processing will earn important value to pattern classification.

In this paper, we consider a decomposition policy of two-class classification task, which is one of two processing stages in min-max modular $\left(\mathrm{M}^{3}\right)$ neural network[1]-[2]. The policy is based on partition of training set, which make it possible that a large-scale two-class problem is divided into some smaller scale two-class subproblems for parallel processing.

However, one hand, it is obvious that such partition will yield much many modules, which cause serious performance problems. On the other hand, only partial sub-classifier modules will actually function for each input sample in classification. This activates us that it is possible to optimize the original combination procedure of classifiers only to check those necessary modules, instead of all produced sub-classifier modules for an input sample. This is to say, an additional module selection procedure can be introduced for improving the response performance of the classifier.

In this paper, we will present an efficient module selecting policy named symmetrical selection algorithm to improve the response performance of minmax modular classifier.

\footnotetext{
* This work was supported in part by the National Natural Science Foundation of China via the grants NSFC 60375022 and NSFC 60473040.
} 


\section{Task Decomposition and Module Combination}

Consider a two-class classification problem, whose output coding of class labels are denoted by $C_{0}$ and $C_{1}$ (as two values, $C_{1}>C_{0}$ ), or equally, 0 and 1 , which will be concise and not lose any generality for our algorithm description. Suppose the training set of class $C_{0}$ is decomposed into $n$ subsets and the training set of class $C_{1}$ is divided into $m$ subsets. By arranging those $m$ and $n$ training subsets in pairs, we obtain $m \times n$ training set pairs. Each pair is learned by a single binary sub-classifier. Therefore, a larger scale two-class problem can be decomposed into $m \times n$ smaller subproblems. We call the binary sub-classifier as base classifier.

Suppose all produced training set pairs are denoted by $X_{i j}$, for $i=0,1, \ldots$, $m-1$, and $j=0,1, \ldots, n-1$. Without misunderstanding, we also express $X_{i j}$ as the output of the corresponding base classifier.

Min-max combination defines how the outputs of those $m \times n$ base classifiers are combined into the solution to the original problem. Before combination, a grouping operation on $m \times n$ base classifiers should be done: these base classifiers, $X_{i j}$, for $j=0, \ldots, n-1$, are defined as one " $C_{1}$ group" and $i$ is defined as its group label, and those base classifiers, $X_{i j}$, for $i=0, \ldots, m-1$, are defined as one " $C_{0}$ group" and $j$ is defined as its group label.

Min-max combination of all base classifiers includes two stages: Firstly, the minimization rule is applied to each $C_{1}$ group to make the output of the group. Secondly, the outputs of all groups are integrated by the maximization rule to make the final output of the original two-class classification problem.

An outstanding merit for min-max modular classifier is that imbalanced classification can always be avoid from a simple decomposition if needed[4].

\section{Symmetrical Module Selection Algorithm}

We call a $C_{1}$ group whose all member base classifiers hold the class $C_{1}$ as a "winning group of class $C_{1}$ " and a class $C_{0}$ group whose all member base classifiers hold the class $C_{0}$ as a "winning group of class $C_{0}$ " . It is easy to demonstrate that in the original min-max combination procedure for one input sample, the fact that there is a winning group of $C_{1}$ in all $m \times n$ base classifiers will be sufficient and necessary to make the conclusion that the final combination result must be class $C_{1}$ according to the combining rules, vice versa, if any winning group of $C_{1}$ does not exist, then the final combination result must be class $C_{0}$. Thus, a min-max combination of base classifiers will be redescribed as such a search procedure to find a winning group of class $C_{1}$.

Similarly, we can also define one symmetrical max-min combination to perform a search procedure for a winning group of class $C_{0}$. Naturally, that there exists a winning group of class $C_{0}$ in all $m \times n$ base classifiers is a sufficient and necessary criterion for a combination result of class $C_{0}$. Symmetrical module selection algorithm, just like its name, is a procedure with consideration of the winning group of both class $C_{0}$ and class $C_{1}$, instead of one-sided search for the winning group of class $C_{1}$ in the original model[1],[2]. 
Basically, symmetrical module selection is based on the following idea: a) Notice that it is impossible that there exists one winning group of class $C_{1}$ and one winning group of class $C_{0}$ at the same time. The reason is that any winning group of class $C_{1}, X_{i j}$, for $j=0, \ldots, n-1$, and any winning group of class $C_{0}$, $X_{i j}$, for $i=0, \ldots, m-1$, must share one same base classifier, which can not output two different classification results at the same time. b) The symmetrical module selection algorithm is a procedure to continuously exclude those hopeless class $C_{1}$ or $C_{0}$ group. The existence of a winning group of class $C_{0}$ or class $C_{1}$ means final combination classification result is class $C_{0}$ or class $C_{1}$, respectively. Regarding all outputs of base classifiers as a binary matrix, a row can stand for a class $C_{1}$ group, and a column can stand for a class $C_{0}$ group. Thus, that a base classifier outputs 1 as classification result means that the column it locates loses the chance to be a winning group of class $C_{0}$. Symmetrically, that a base classifier outputs 0 as classification result means that the column it locates loses the chance to be a winning group of class $C_{1}$.

The symmetrical module selection algorithm is described below in detail. For convenience, we also say the first subscript of a base classifier is its row, and the second is its column, also a row means a class $C_{1}$ group and a column means a class $C_{0}$ group.

1. For $i=0, \ldots, m-1$, set the $\operatorname{tag} R F[i]=0$, and for $j=0, \ldots, n-1$, set the $\operatorname{tag} C F[j]=0$, which means all class one and zero groups are not excluded, respectively.

2. Set two counters, RCounter $=m$ and CCounter $=n$, respectively, which means the number of surviving class one and zero groups without excluded.

3. Set starting row and column, $i=0$ and $j=0$.

4. Repeat the following operations:

(a) If $i$ is an excluded group label, that is, $R F[i]=1$, then $i$ increases continuously until $i$ is not an excluded label any more or $i=m$.

(b) If $j$ is an excluded group label, that is, $C F[j]=1$, then $j$ increases continuously until $j$ is not an excluded label any more or $j=n$.

(c) If the counter, RCounter $=0$, or $i=m$, then output the combination classification result class 0 and the algorithm ends here.

(d) If the counter, CCounter $=0$, or $j=n$, then output the combination classification result class 1 and the algorithm ends here.

(e) Check the base classifier $X_{i j}$.

(f) If $X_{i j}$ holds class $C_{1}$, then

i. Set the tag, $C F[j]=1$.

ii. Subtract 1 from the counter, CCounter $=$ CCounter -1

iii. $j=j+1$.

(g) If $X_{i j}$ holds class $C_{0}$, then

i. Set the tag, $R F[i]=1$.

ii. Subtract 1 from the counter, $R$ Counter $=$ RCounter -1

iii. $i=i+1$. 


\section{Performance Estimation}

We now show that the number of checked base classifiers in symmetrical module selection algorithm for each input sample will be never larger than $m+n-1$.

In fact, the algorithm is equally a search procedure in a binary matrix with $m$ rows and $n$ columns, in which the start point is top left corner and the end point is bottom right corner. Each checking for a base classifier is equal to an access for one element in the matrix, which means one row or one column in the matrix must be excluded. In turn, the accessing of the algorithm gets to the next row or column without any backtracking. In summary, such search from top left corner to bottom right corner in this matrix mostly covers $m+n-1$ elements. Namely, the number of checked base classifier in symmetrical module selection for each input sample will not be larger than $m+n-1$, which is much better than the case, $m \times n$, under the original model.

\section{Experimental Results}

Three data sets shown in Table 1 from UCI Repository[3] have been chosen for this study. For a typical realization of min-max modular classifier, support vector machine(SVM) with RBF kernel is selected as base classifier[4]. Two parameters, $C$ and $\gamma$, are set to 8 and 0.25 for Internet Ads and Adult data sets, respectively. These two parameters are set to 316.2 and 1 for Banana data set, respectively. The same numbers of samples for class $C_{1}$ and $C_{0}$ in every subsets are taken.

Table 1. Distributions of samples in three data sets

\begin{tabular}{c|c|c|c|c|c|c}
\hline \hline \multirow{2}{*}{ Data Set } & \multicolumn{2}{|c|}{ Number of Training Samples } & \multicolumn{3}{|c}{ Number of Test Samples } \\
\cline { 2 - 7 } & Total & Class $C_{1}$ & Class $C_{0}$ & Total & Class $C_{1}$ & Class $C_{0}$ \\
\hline Internet Ads & 2100 & 1800 & 300 & 1179 & 1020 & 159 \\
\hline Adult & 32561 & 24720 & 7841 & 16281 & 12435 & 3846 \\
\hline Banana & 40000 & 21847 & 18153 & 490000 & 270553 & 219447 \\
\hline
\end{tabular}

Experimental results are shown in Tables 2 through 4 . The comparison between experimental results and theoretical bounds in three data sets are shown in Fig 1. Fig 1(a) shows 20 different experimental results, where $n$ varies from 2 to 21 and $m$ correspondingly varies from 12 to 129 for Internet Ads data set. Fig 1(b) shows 100 different experimental results, where $n$ varies from 2 to 101 and $m$ correspondingly varies from 6 to 317 for Adult data set. Note that Table 2 and Table 3 only show parts of experimental results shown in Fig.1(a) and Fig.1(b) for space limitation.

As expected, the experimental results show that our symmetrical module selection algorithm gives an outstanding performance improvement for testing procedure with respect to the case without any selection, while combining accuracies keep the same. The number of actual checked base classifier is also strictly 
Table 2. Experimental results on Internet Ads data set

\begin{tabular}{c|c|c|c|c|c|c|c|c|c}
\hline \hline \#Modules & \multirow{2}{*}{$\mathrm{m}$} & \multirow{n}{*}{$\mathrm{n}$} & \multicolumn{3}{|c|}{ Without Selection } & \multicolumn{4}{c}{ Symmetrical Selection } \\
\cline { 5 - 10 } & & & \#Modules & Time(ms) & acc.(\%) & \#Modules & Bound & Time(ms) & acc.(\%) \\
\hline 14 & 129 & 21 & 2709 & 63656 & 90.16 & 29.04 & 149 & 26718 & 90.16 \\
\hline 18 & 100 & 17 & 1700 & 44046 & 89.40 & 19.78 & 116 & 15484 & 89.40 \\
\hline 23 & 78 & 13 & 1014 & 29890 & 89.31 & 15.07 & 90 & 8265 & 89.31 \\
\hline 33 & 56 & 9 & 504 & 17859 & 89.57 & 10.62 & 64 & 3375 & 89.57 \\
\hline 60 & 30 & 5 & 150 & 8421 & 89.90 & 5.97 & 34 & 1000 & 89.90 \\
\hline
\end{tabular}

Table 3. Experimental results on Adult data set

\begin{tabular}{c|c|c|c|c|c|c|c|c|c}
\hline \hline \#Modules & \multirow{2}{*}{$\mathrm{m}$} & \multirow{2}{*}{$\mathrm{n}$} & \multicolumn{3}{|c|}{ Without Selection } & \multicolumn{3}{c}{ Symmetrical Selection } \\
\cline { 4 - 10 } & & & \#Modules & Time(ms) & acc.(\%) & \#Modules & Bound & Time(ms) & acc.(\%) \\
\hline 320 & 77 & 25 & 1925 & 7027 & 76.37 & 25.45 & 101 & 116 & 76.37 \\
\hline 157 & 157 & 50 & 7850 & 15085 & 76.36 & 96.79 & 206 & 281 & 76.36 \\
\hline 105 & 235 & 75 & 17625 & 22613 & 76.39 & 183.19 & 309 & 430 & 76.39 \\
\hline 77 & 321 & 102 & 32742 & 37679 & 76.37 & 320.97 & 422 & 666 & 76.37 \\
\hline \hline
\end{tabular}

Table 4. Experimental results on Banana data set

\begin{tabular}{c|c|c|c|c|c|c|c|c|c}
\hline \hline \#Modules & \multirow{2}{*}{$\mathrm{m}$} & \multirow{2}{*}{$\mathrm{n}$} & \multicolumn{3}{|c|}{ Without Selection } & \multicolumn{4}{c}{ Symmetrical Selection } \\
\cline { 4 - 10 } & & & \#Modules & Time $(\mathrm{ms})$ & acc.(\%) & \#Modules & Bound & Time(ms) & acc.(\%) \\
\hline 90 & 243 & 202 & 49086 & 154023 & 90.81 & 229.56 & 444 & 1389 & 90.81 \\
\hline 103 & 212 & 176 & 37312 & 130945 & 90.73 & 199.20 & 387 & 1279 & 90.73 \\
\hline 121 & 181 & 150 & 27150 & 107417 & 90.99 & 169.43 & 330 & 1096 & 90.99 \\
\hline 145 & 151 & 125 & 18875 & 88564 & 90.68 & 141.66 & 275 & 883 & 90.68 \\
\hline 181 & 121 & 100 & 12100 & 66653 & 90.61 & 112.55 & 220 & 754 & 90.61 \\
\hline 242 & 90 & 75 & 6750 & 52709 & 90.80 & 84.01 & 164 & 679 & 90.80 \\
\hline 363 & 60 & 50 & 3000 & 32309 & 90.75 & 55.87 & 109 & 645 & 90.75 \\
\hline \hline
\end{tabular}

under the theoretical bound. However, the ratio of numbers of checked modules and the ratio of practical response time between two cases are not exactly the same, which may mostly attribute to different number of support vectors obtained by each base classifier.

\section{Conclusions}

An efficient module selection policy has been presented for improving the response performance of min-max modular classifier. We show that the quadratic 


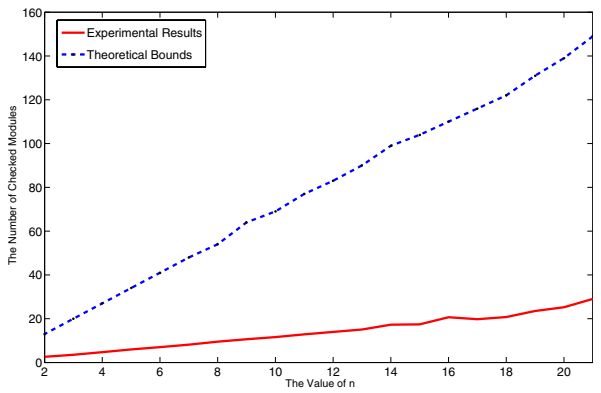

(a)

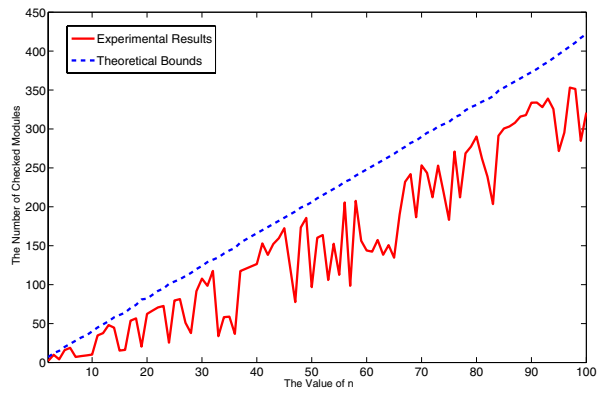

(b)

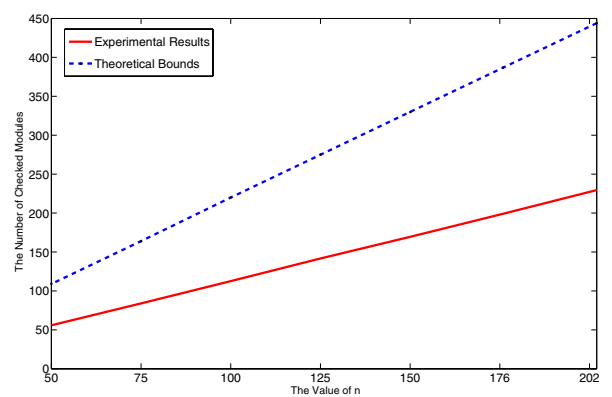

(c)

Fig. 1. Comparison of theoretical bound and experimental result on Internet Ads data set(a), Adult data set(b), and Banana data set(c)

complexity of the original min-max modular classifier can be reduced onto linear complexity of the number of base-classifiers for each input sample. The experimental results indicate that an outstanding improvement on the response performance for such modular classifier is obtained.

\section{References}

1. Lu, B.L., Ito, M.: Task Decomposition Based on Class Relations: a Modular Neural Network Architecture for Pattern Classification. Lecture Notes in Computer Science. Vol. 1240. Springer (1997) 330-339

2. Lu, B.L., Ito, M.: Task Decomposition and Module Combination Based on Class Relations: a Modular Neural Network for Pattern Classification. IEEE Transactions on Neural Networks, 10 (1999) 1244-1256

3. Blake, C.L., Merz, C.J: UCI Repository of Machine Learning Databases [http://www.ics.uci.edu/ mlearn/MLRepository.html]. Irvine, CA: University of California, Department of Information and Computer Science (1998)

4. Lu, B.L., Wang, K. A., Utiyama, M., Isahara, H.: A Part-versus-part Method for Massively Parallel Training of Support Vector Machines. Proc. of IEEE/INNS Int. Joint Conf. on Neural Networks ( IJCNN2004), Budabest, Hungary (2004) 25-29 